Article

\title{
Electrical and Hormonal Biomarkers in Cachectic Elderly Women with Chronic Heart Failure
}

\author{
Grzegorz Sobieszek ${ }^{1, *}$, Tomasz Powrózek ${ }^{2, *}$, Marcin Mazurek ${ }^{2}$, Anna Skwarek-Dziekanowska ${ }^{1}$ \\ and Teresa Małecka-Massalska ${ }^{2}$ \\ 1 Department of Cardiology, 1st Military Clinical Hospital with the Outpatient Clinic, 20-048 Lublin, Poland; \\ anetask@gmail.com \\ 2 Department of Human Physiology, Medical University of Lublin, 20-080 Lublin, Poland; \\ marcinmazurek1212@gmail.com (M.M.); t.malecka@gmail.com (T.M.-M.) \\ * Correspondence: grzes.bies@interia.pl (G.S.); tomaszpowrozek@gmail.com (T.P.); \\ Tel.: +48-261183614 (G.S.); +48-814486080 (T.P.)
}

Received: 7 March 2020; Accepted: 2 April 2020; Published: 4 April 2020

\begin{abstract}
Background: Cachexia is an unfavorable metabolic syndrome causing involuntary weight loss followed by muscle wasting, which can lead to the exacerbation of chronic heart failure (CHF), and considerably increases mortality rate among CHF patients. Unfortunately, until now it has not been possible to determine factors that could improve clinical options for cachexia management or enable the identification of patients at risk of its development. We assessed how cachexia conditions in $\mathrm{CHF}$ reflect cardiac and laboratory parameters in comparison with non-cachectic patients. Methods: 66 women were enrolled to the study group and underwent meticulous screening, according to recent clinical guidelines, in order to enable CHF and cachexia detection. Body composition was evaluated by bioelectrical impedance analysis (BIA) and laboratory tests were supplemented by analysis of plasma circulating irisin. Results: A negative correlation between irisin concentration and both CRP and TNF- $\alpha$ was recorded $(R=-0.362$ and $R=-0.243 ; p<0.05)$. Irisin concentration positively correlated with EF\% $(R=0.253 ; p=0.046)$ and negatively with LVESd, LVEDd and NT-proBNP $(R=-0.326,-0.272$, and $-0.320 ; p<0.05)$. Both low levels of circulating irisin and Capacitance of membrane $(\mathrm{Cm})$ were selected as unfavorable factors affecting cachexia in $\mathrm{CHF}$ patients $(\mathrm{OR}=1.39$ and 34.49; $p<0.05)$. Combination of $\mathrm{Cm}$, irisin, CRP and albumin demonstrated sensitivity of $93.3 \%$ and specificity of $85.3 \%$ (AUC $=0.949$ ) for distinguishing between cachectic and non-cachectic $\mathrm{CHF}$ patients. Conclusions: Selected parameters reliably reflect cachectic conditions in $\mathrm{CHF}$, and the proposed approach for cachexia based on the combined analysis of at least a few non-invasive markers could offer new opportunities for improving clinical outcomes in CHF patients.
\end{abstract}

Keywords: chronic heart failure; cachexia; irisin; bioimpedance analysis; biomarker

\section{Introduction}

Chronic heart failure (CHF) is a disease with growing incidence in adults in highly developed countries, and itsrisk factors include presence of other cardiovascular diseases or the coexistence of chronic diseases [1,2]. Due to metabolic alterations associated with the disease, CHF patients are increasingly diagnosed with heart failure-induced cachexia (cardiac cachexia; CC), which is diagnosed in $8-42 \%$ CHF patients, and the mortality rate is roughly $20-30 \%$. Progression of cachexia in CHF patients is based on the reduced myocardial perfusion, cardiomyocyte decline and the depletion of high energy stores (reduced cardiac function and increased catabolic state of the body) [3-5]. Until now, there is lack of literature reports concerning combined use of hormonal markers (e.g., irisin) and other tools (bioelectrical impedance analysis, BIA) in the management of cachexia in patients with CHF. 
Irisin, a thermogenic adipomyokine, regulates body energy metabolism and then its conversion in the form of ATP, but also participates in the browning of adipose tissue [6,7]. The blood concentration of irisin is significantly altered in patients suffering from acute coronary syndrome, especially in myocardial infarction (AMI), ST-Elevation Myocardial Infarction (STEMI) and stable coronary disease. Irisin seems to be a clinically promising marker reflecting cardiovascular system efficiency and the metabolic condition of the entire body of patients with CHF [8,9]. BIA as a non-invasive method reliably evaluates body composition and its nutritional status at the cellular level. The two potentially clinically useful parameters, which can be obtained from BIA, are membrane capacitance $(\mathrm{Cm})$ and phase angle (PA). They show clinical usefulness in the body composition assessment and the detection of malnutrition and demonstrate a prognostic value in various diseases accompanied by malnutrition, including CHF [1,10-12].

The aim of the study was to assess how cachexia conditions in CHF females reflects cardiac and laboratory parameters in comparison with non-cachectic patients. Our study is limited only to women, in order to be able to interpret the electrical, hormonal and biochemical changes. Age, sex and body mass index are the determinants of the electrical parameters.

\section{Patients and Methods}

\subsection{Study Group}

Sixty-six women (mean age: $77 \pm 9$ years) with newly diagnosed CHF were enrolled to the study group. All study participants were inpatients diagnosed and treated at the Clinic of Cardiology and Internal Medicine, Department of Cardiology, Military Hospital in Lublin, Poland between 2017 and 2019. CHF was diagnosed according to the recent European Society of Cardiology (ECS) criteria, that are based on a patient's clinical evaluation and echocardiographic assessment supplemented by blood examination, including a serum concentration of $\mathrm{N}$-terminal prohormone of brain natriuretic peptide (NT-proBNP), lipid profile, creatinine and hemoglobin level [13]. The extent of the disease was classified according to New York Association (NYHA) guidelines, which qualify patients to four functional classes: I-IV based on the symptom's severity [14]. For the study protocol, the defined inclusion and exclusion criteria were applied. The inclusion criteria to the study were as follows: (1) age $>18$ years, female gender and Polish ethnicity; (2) signed consent to participate in the study protocol; (3) newly diagnosed CHF. The exclusion criteria were as follows: (1) extreme renal failure or eGFR $<15 \mathrm{~mL} / \mathrm{min} / 1,73 \mathrm{~m}^{2}$; (2) acute coronary syndrome; (3) a coronary artery bypass grafting within the last 6 months; (4) hyper- or hypothyroidism; (5) presence of a heart pacemaker, cardioverter or defibrillator, as well as a lack of metallic implants. Detailed baseline characteristics of the study group is presented in Table 1.

Table 1. Characteristics of the study group (ACEi-Angiotensin-converting-enzyme inhibitors; ARBs—angiotensin receptor blockers; BMI—body mass index; $\mathrm{Cm}$-capacitance of membrane; EF-ejection fraction; FM-fat mass; FFM—fat-free mass; HRT-Hormone replacement therapy; LAD—left anterior descending artery; LVEDd—left ventricular end-diastolic diameter; LVESd—left ventricular end-systolic diameter; NYHA—New York Heart Association; Pa—phase angle (50kHz); PASP — pulmonary artery systolic pressure; RVOT—right ventricular outflow tract; SGA—subjective global assessment; TAPSE-tricuspid annular piane systolic excursion) ${ }^{+}$-non-parametric Mann-Whitney $U$-test; the other parameters were tested by parametric student's $t$-test.

\begin{tabular}{cc}
\hline Factor & Study Group $(\boldsymbol{n}=\mathbf{6 6})$ \\
\hline Age (years) & $77 \pm 9.0$ \\
\hline Weight $(\mathrm{kg})$ & $77 \pm 18.0$ \\
\hline BMI $\left(\mathrm{kg} / \mathrm{m}^{2}\right)$ & $29.75 \pm 6.52$ \\
\hline
\end{tabular}


Table 1. Cont.

\begin{tabular}{|c|c|c|}
\hline \multicolumn{2}{|c|}{ Factor } & Study Group $(n=66)$ \\
\hline \multicolumn{2}{|c|}{$\mathrm{FM}(\mathrm{kg})$} & $26.45 \pm 11.14$ \\
\hline \multicolumn{2}{|c|}{ FFM (kg) } & $50.08 \pm 12.45$ \\
\hline \multicolumn{2}{|c|}{ Albumin (g/dL) } & $3.44 \pm 0.59$ \\
\hline \multicolumn{2}{|c|}{ Triglycerides (mg/dL) } & $119.8 \pm 64.0$ \\
\hline \multicolumn{2}{|c|}{ Total cholesterol (mg/dL) } & $172.1 \pm 46.2$ \\
\hline \multicolumn{2}{|c|}{ HDL (mg/dL) } & $54.66 \pm 16.9$ \\
\hline \multicolumn{2}{|c|}{$\mathrm{LDL}(\mathrm{mg} / \mathrm{dL})$} & $91.80 \pm 36.79$ \\
\hline \multicolumn{2}{|c|}{ Creatinine (mg/dL) } & $1.19 \pm 0.53$ \\
\hline \multicolumn{2}{|c|}{ Hemoglobin (g/dL) } & $12.48 \pm 1.93$ \\
\hline \multicolumn{2}{|c|}{$\mathrm{CRP}(\mathrm{mg} / \mathrm{L})^{\dagger}$} & $4.0(1.60-17.43)$ \\
\hline \multicolumn{2}{|c|}{$\mathrm{TNF}-\alpha(\mathrm{pg} / \mathrm{mL})^{\dagger}$} & $3.92(3.22-5.10)$ \\
\hline \multicolumn{2}{|c|}{ IL-6 (pg/mL) } & $5.52(3.21-8.69)$ \\
\hline \multicolumn{2}{|c|}{$\operatorname{Irisin}(\mu \mathrm{g} / \mathrm{mL})^{+}$} & $7.12(5.70-9.99)$ \\
\hline \multicolumn{2}{|c|}{ Systolic blood pressure (mmHg) } & $134 \pm 22.0$ \\
\hline \multicolumn{2}{|c|}{ Diastolic blood pressure (mmHg) } & $76 \pm 11.0$ \\
\hline \multicolumn{2}{|c|}{$\mathrm{EF} \%$} & $45 \pm 11.0$ \\
\hline \multicolumn{2}{|c|}{ NT-proBNP $(\mathrm{pg} / \mathrm{mL})^{\dagger}$} & $2332(1002-4010)$ \\
\hline \multicolumn{2}{|c|}{ LVESd (cm) } & $4.04 \pm 0.83$ \\
\hline \multicolumn{2}{|c|}{ LVEDd (cm) } & $5.45 \pm 0.79$ \\
\hline \multicolumn{2}{|c|}{$\operatorname{LAD}(\mathrm{cm})$} & $4.45 \pm 0.63$ \\
\hline \multicolumn{2}{|c|}{ RVOT $(\mathrm{cm})$} & $3.33 \pm 0.37$ \\
\hline \multicolumn{2}{|c|}{ TAPSE $(\mathrm{cm})$} & $1.91 \pm 0.40$ \\
\hline \multicolumn{2}{|c|}{ PASP (mmHg) } & $41 \pm 11.0$ \\
\hline \multirow{4}{*}{ NYHA } & $\mathrm{I}$ & $13(19.7 \%)$ \\
\hline & II & $21(31.8 \%)$ \\
\hline & III & $21(31.8 \%)$ \\
\hline & IV & $11(16.7 \%)$ \\
\hline \multirow{3}{*}{ SGA } & A & $35(53 \%)$ \\
\hline & B & $23(34.8 \%)$ \\
\hline & $\mathrm{C}$ & $8(12.2 \%)$ \\
\hline \multicolumn{2}{|c|}{ Diabetes mellitus } & $28(42.4 \%)$ \\
\hline Renal & ailure & $25(37.9 \%)$ \\
\hline ACEi t & atment & $40(60.6 \%)$ \\
\hline ARBs $t$ & atment & $11(16.7 \%)$ \\
\hline HRT tr & atment & $8(12.1 \%)$ \\
\hline SmokingStatus & smoker & $36(54.5 \%)$ \\
\hline & non-smoker & $30(45.5 \%)$ \\
\hline $\mathrm{Cm}$ & F) ${ }^{\dagger}$ & $1.159(0.750-1.681)$ \\
\hline & & $4.09 \pm 1.22$ \\
\hline $\mathrm{Z2C}$ & Z5 & $0.853(0.830-0.879)$ \\
\hline
\end{tabular}


Study protocol was approved by Bioethical Commission in Medical University of Lublin (no of consent: KE-0254/64/2017). Prior to the study, all patients signed informed consent forms.

\subsection{Nutritional Assessment and Cachexia Detection}

Cachexia was diagnosed according to the criteria proposed by Evans WJ et al: a weight loss of at least $5 \%$ or more in 12 months or less in the presence of underlying illness, plus three of the following criteria: decreased muscle strength, fatigue, anorexia, low fat-free mass index, abnormal biochemistry (increased inflammatory markers: C-reactive protein $>5.0 \mathrm{mg} / \mathrm{L}), \mathrm{IL}-6>4.0 \mathrm{pg} / \mathrm{mL})$, anemia $(<12 \mathrm{~g} / \mathrm{dL}$ ) and low level of serum albumin $(<3.2 \mathrm{~g} / \mathrm{dL}))$ [15].

\subsection{Bioelectrical Impedance Analysis}

BIA measurement was conducted with the use of an ImpediMed bioimpedance analysis SFB7 BioImp v1.55 device (Pinkenba, Queensland, Australia). To provide reliable measurements, we applied similar BIA conditions for all study participants-the data was collected in the morning and on an empty stomach. Prior to examination, patients were lying in a bed in the supine position (lying on the back and their legs and arms were not in contact with the torso) for at least five minutes, to equalize a body fluid level. All measurements were performed on the right side of the body (right-side location of the electrodes). Cm, Pa, Z5 and Z200 values were automatically obtained from the BIA equipment. Then the Z200/Z5 ratio was calculated. The following parameters reflecting body composition of the patients were derived from the BIA: fat mass (FM) and fat-free mass (FFM).

\subsection{Plasma IrisinConcentration}

From each study participant, $5 \mathrm{~mL}$ of whole blood was collected in order to assess plasma irisin level. After plasma separation, samples were stored at $-80^{\circ} \mathrm{C}$ until the analysis. Plasma irisin level was measured according to the manufacturer's protocol, with the use of the dedicated Irisin ELISA Kit (BioVendor, Brno, Czech Republic). The detection range of the kit was 0.001-5 $\mu \mathrm{g} / \mathrm{mL}$, and the sensitivity was equal to the minimal detectable dose of this kit $(<1 \mathrm{ng} / \mathrm{mL})$.

\subsection{Statistical Analysis}

Statistical analysis was conducted with the use of MedCalc computer software (version 15.3) (MedCalc, Ostend, Belgium). All graphs illustrating results of the statistical analysis were generated by the same applied statistical software. Data distribution of the collected variables was checked by the Shapiro-Wilk test. Based on the result of data distribution analysis, either parametric student's $t$-test or non-parametric Mann-Whitney $U$-test were applied to compare anthropometric, biochemical, nutritional and cardiac parameters between the cachectic and non-cachectic group of HF patients. Correlation between irisin and studied parameters was tested with the use of Spearman's rank correlation. The uni- and multivariate logistic regression model with the odds ratio calculation (OR) was applied to select factors affecting the cachexia incidence in female CHF individuals. Receiver operating curves (ROC) with area under the curve (AUC) calculation were used to assess accuracy of the selected parameters for distinguishing between cachectic and non-cachectic CHF patients. Results with $p$ values below 0.05 were considered as being statistically significant.

\section{Results}

Median concentration of plasma irisin was significantly decreased in cachectic women compared with non-cachectic ones (median irisin concentration: $7.12 \mu \mathrm{g} / \mathrm{mL}$ (IQR: 5.94-9.42) and $7.61 \mu \mathrm{g} / \mathrm{mL}$ (IQR: 5.29-10.39); $p=0.022$ ). Moreover, increased level of inflammatory markers was noted in cachectic patients compared to women without symptoms of cachexia (median CRP level: $10.95 \mathrm{mg} / \mathrm{L}$ (1.96-26.20) and $3.10 \mathrm{mg} / \mathrm{L}$ (1.45-4.95); $p=0.005$; median TNF- $\alpha$ level: $4.48 \mathrm{pg} / \mathrm{mL}$ (3.49-5.13) and $3.29 \mathrm{pg} / \mathrm{mL}$ (3.07-4.91); $p=0.032$ ). Regarding parameters reflecting cardiac function, we recorded significant 
differences between analyzed groups. First of all, cachectic patients demonstrated reduced EF\% (mean: $42 \pm 13 \%$ and $48 \pm 9 \% ; p=0.039$ ) and a significantly higher level of NT-proBNP (median: $3476 \mathrm{pg} / \mathrm{mL}$ (IQR:1690-5773) and $1176 \mathrm{pg} / \mathrm{mL}$ (IQR:716-2605); $p<0.001$ ). Severe cardiac symptoms defined as NYHA III and IV class were more frequent among cachectic patients. Some $61.8 \%$ of women belonging to cachectic group were qualified as either NYHA III or IV class, while the aforementioned proportion was $34.4 \%$ in non-cachectic patients $(p=0.030)$. All of the following parameters reflecting the nutritional status of CHF patients—body weight, BMI, FM, FFM and albumin -were significantly reduced in the cachexia group $(p<0.05)$. Also, the significant differences in BIA parameters reflecting the nutritional status of the body cells were observed among patients. The most significant difference between cachectic and non-cachectic patients was recorded for the $\mathrm{Cm}$ (median $\mathrm{Cm}$ : $0.860 \mathrm{nF}$ (IQR: $0.670-1.070$ ) and 1.280 nF (IQR: 0.916-1.789); $p<0.001$ ). Differences in studied parameters between cachectic and non-cachectic patients are summarized in Table 2.

Table 2. Differences in anthropometric, metabolic, inflammatory, nutritional and cardiac parameters between chronic heart failure (CHF) patients with either presence or absence of cachexia (ACEi-Angiotensin-converting-enzyme inhibitors; ARBs-angiotensin receptor blockers; BMI—body mass index; $\mathrm{Cm}$-capacitance of membrane; EF-ejection fraction; FM-fat mass; FFM-fat-free mass; HRT—Hormone replacement therapy; LAD—left anterior descending artery; LVEDd—left ventricular end-diastolic diameter; LVESd—left ventricular end-systolic diameter; NYHA-New York Heart Association; Pa—phase angle (50kHz); PASP—pulmonary artery systolic pressure; RVOT—right ventricular outflow tract; SGA—subjective global assessment; TAPSE—-tricuspid annular piane systolic excursion) ${ }^{\dagger}$-non-parametric Mann-Whitney $U$-test; the other parameters were tested by parametric student's $t$-test.

\begin{tabular}{|c|c|c|c|}
\hline Factor & Cachectic $(n=34)$ & Non-Cachectic $(n=32)$ & $p$ \\
\hline Age (years) & $80 \pm 12$ & $77 \pm 9$ & 0.267 \\
\hline Weight (kg) & $71 \pm 17$ & $82 \pm 17$ & 0.010 \\
\hline BMI $\left(\mathrm{kg} / \mathrm{m}^{2}\right)$ & $28.02 \pm 6.22$ & $31.64 \pm 6.41$ & 0.024 \\
\hline $\mathrm{FM}(\mathrm{kg})$ & $23.14 \pm 9.32$ & $29.92 \pm 10.89$ & 0.020 \\
\hline FFM (kg) & $46.61 \pm 13.21$ & $53.96 \pm 10.48$ & 0.031 \\
\hline Albumin (g/dL) & $3.14 \pm 0.61$ & $3.76 \pm 0.36$ & $<0.001$ \\
\hline Triglycerides (mg/dL) & $118.0 \pm 60.6$ & $121.9 \pm 68.6$ & 0.806 \\
\hline Total cholesterol (mg/dL) & $167.6 \pm 52.0$ & $177.0 \pm 39.0$ & 0.417 \\
\hline HDL (mg/dL) & $49.90 \pm 16.72$ & $59.88 \pm 15.94$ & 0.017 \\
\hline LDL (mg/dL) & $92.26 \pm 39.88$ & $91.28 \pm 33.72$ & 0.915 \\
\hline Hemoglobin (g/dL) & $11.97 \pm 1.84$ & $13.04 \pm 1.89$ & 0.025 \\
\hline $\mathrm{CRP}(\mathrm{mg} / \mathrm{L})^{\dagger}$ & $10.95(1.96-26.20)$ & $3.10(1.45-4.95)$ & 0.005 \\
\hline TNF- $\alpha(p g / m L)^{\dagger}$ & $4.48(3.49-5.13)$ & $3.29(3.07-4.91)$ & 0.032 \\
\hline $\operatorname{Irisin}(\mu \mathrm{g} / \mathrm{mL})^{\dagger}$ & $7.12(5.94-9.42)$ & $7.61(5.29-10.39)$ & 0.022 \\
\hline Systolic blood pressure (mmHg) & $132 \pm 22$ & $137 \pm 22$ & 0.409 \\
\hline Diastolic blood pressure (mmHg) & $75 \pm 12$ & $76 \pm 11$ & 0.754 \\
\hline $\mathrm{EF} \%$ & $42 \pm 13.0$ & $48 \pm 9.0$ & 0.039 \\
\hline NT-proBNP $(\mathrm{pg} / \mathrm{mL})^{\dagger}$ & $3476(1690-5773)$ & $1176(716-2605)$ & $<0.001$ \\
\hline LVESd (cm) & $4.11 \pm 0.89$ & $3.97 \pm 0.80$ & 0.514 \\
\hline
\end{tabular}


Table 2. Cont.

\begin{tabular}{|c|c|c|c|c|}
\hline \multicolumn{2}{|c|}{ Factor } & Cachectic $(n=34)$ & Non-Cachectic $(n=32)$ & $p$ \\
\hline \multicolumn{2}{|c|}{ LVEDd $(\mathrm{cm})$} & $4.99 \pm 0.85$ & $5.04 \pm 0.71$ & 0.833 \\
\hline \multicolumn{2}{|c|}{ LAD (cm) } & $4.54 \pm 0.72$ & $4.36 \pm 0.52$ & 0.271 \\
\hline \multicolumn{2}{|c|}{ RVOT (cm) } & $3.35 \pm 0.41$ & $3.32 \pm 0.32$ & 0.704 \\
\hline \multicolumn{2}{|c|}{ TAPSE $(\mathrm{cm})$} & $1.84 \pm 0.42$ & $1.96 \pm 0.37$ & 0.180 \\
\hline \multicolumn{2}{|c|}{ PASP (mmHg) } & $42.8 \pm 12.5$ & $39.2 \pm 10.0$ & 0.208 \\
\hline \multicolumn{2}{|c|}{ NYHA I+II } & $13(38.2 \%)$ & $21(65.6 \%)$ & \multirow{2}{*}{0.030} \\
\hline \multicolumn{2}{|c|}{ NYHA III+IV } & $21(61.8 \%)$ & $11(34.4 \%)$ & \\
\hline \multirow{2}{*}{ ACEi } & Yes & $18(52.9 \%)$ & $22(68.8 \%)$ & \multirow{2}{*}{0.216} \\
\hline & No & $16(47.1 \%)$ & $10(31.2 \%)$ & \\
\hline \multirow{2}{*}{$\mathrm{ARBs}$} & Yes & $8(23.5 \%)$ & $3(9.4 \%)$ & \multirow{2}{*}{0.188} \\
\hline & No & $26(76.5 \%)$ & $29(90.6 \%)$ & \\
\hline \multirow{2}{*}{ HRT } & Yes & $1(3 \%)$ & $7(21.9 \%)$ & \multirow{2}{*}{0.025} \\
\hline & No & $33(97 \%)$ & $25(78.1 \%)$ & \\
\hline \multicolumn{2}{|c|}{ SGA-A } & $9(26.5 \%)$ & $26(81.3 \%)$ & \multirow{2}{*}{$<0.001$} \\
\hline \multicolumn{2}{|c|}{ SGA-B+C } & $25(73.5 \%)$ & $6(18.7 \%)$ & \\
\hline \multicolumn{2}{|c|}{$\mathrm{Cm}(\mathrm{nF})^{\dagger}$} & $0.860(0.670-1.070)$ & $1.280(0.916-1.789)$ & $<0.001$ \\
\hline \multicolumn{2}{|c|}{$\mathrm{Pa}\left({ }^{\circ}\right)$} & $3.60 \pm 1.17$ & $4.60 \pm 1.08$ & 0.005 \\
\hline \multicolumn{2}{|c|}{ Z200/Z5 } & $0.877(0.840-0.887)$ & $0.845(0.823-0.854)$ & 0.002 \\
\hline
\end{tabular}

In the subsequent part of the study, we investigated correlation between concentration of plasma irisin in CHF patients, and the studied anthropometric, nutritional and cardiac parameters. The statistically significant results of the correlation study are presented in Table 3.

Table 3. Result of the correlation study-summary of the parameters significantly correlated with plasma irisin level (Cm—capacitance of membrane; EF- ejection fraction; FM—fat mass; LVEDd-left ventricular end-diastolic diameter; LVESd-left ventricular end-systolic diameter).

\begin{tabular}{ccc}
\hline Factor & $\begin{array}{c}\mathbf{R}[95 \% \mathrm{CI}] \\
\text { Positive Correlation }\end{array}$ & $p$ \\
\hline FM & $0.408[0.155$ to 0.681$]$ & 0.020 \\
\hline HDL & $0.318[0.080$ to 0.524$]$ & 0.010 \\
\hline EF\% & $0.253[0.010$ to 0.471$]$ & 0.046 \\
\hline Negative Correlation \\
\hline CRP & $-0.393[-0.580$ to -0.167$]$ \\
\hline LVESd & $-0.362[-0.561$ to -0.123$]$ & 0.005 \\
\hline NT-proBNP & $-0.326[-0.529$ to -0.090$]$ & 0.004 \\
\hline LVEDd & $-0.320[-0.532$ to -0.080$]$ & 0.009 \\
\hline TNF- $\alpha$ & $-0.272[-0.485$ to -0.027$]$ & 0.010 \\
\hline
\end{tabular}

In the whole studied group concentration of plasma irisin significantly correlated with selected parameters reflecting inflammatory, nutritional and cardiac status. We found negative correlation between the level of CRP and TNF- $\alpha$ and irisin; CHF patients with higher irisin concentration had 
reduced inflammatory response represented by CRP and TNF- $\alpha(R=-0.362$ and $R=-0.243 ; p=0.004$ and $p=0.044$, respectively). Correlation between CRP and irisin is shown in Figure 1A. Regarding cardiac parameters, irisin concentration positively correlated with $\mathrm{EF} \%(R=0.253 ; p=0.046)$ and negatively with LVESd, LVEDd and NT-proBNP $(R=-0.326,-0.272$, and $-0.320 ; p=0.009,0.030$, and 0.010 , respectively). Moreover, patients with lower irisin concentration had higher $\mathrm{Cm}$ values $(R=-0.393 ; p=0.005)$ (Figure 1B).
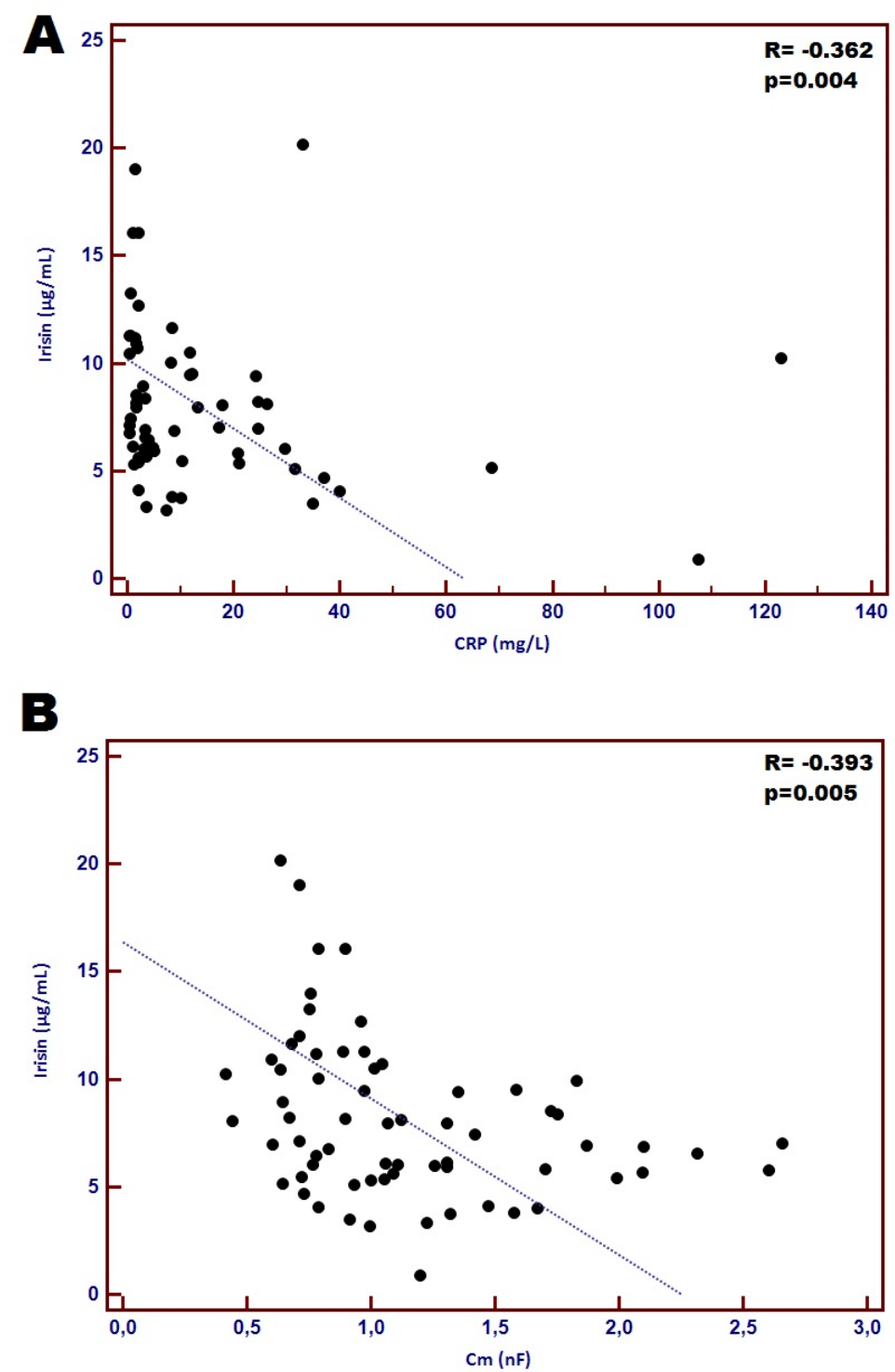

Figure 1. Correlation between concentration of plasma irisin and CRP (A) and correlation between plasma irisin and $\mathrm{Cm}(\mathbf{B})$.

Using logistic regression analysis (uni- and multivariate), the factors significantly affecting the chance of cachexia incidence in CHF patients were identified (Table 4). The univariate analysis revealed albumin concentration and $\mathrm{Cm}$ value as the factors, that most significantly affect probability of cachexia in $\mathrm{CHF}$ patients ( $\mathrm{OR}=33.18$ and $\mathrm{OR}=10.76$, respectively). Similarly, the multivariate analysis model indicates that albumin and $\mathrm{Cm}$ emerge as the independent factors related to cachexia incidence in $\mathrm{CHF}$ women $(\mathrm{OR}=50.48$ and $\mathrm{OR}=34.49$, respectively). 
Table 4. Factors selected by uni- and multivariate logistic regression analysis, that significantly affected cachexia in $\mathrm{CHF}$ female patients.

\begin{tabular}{ccc}
\hline \multicolumn{3}{c}{ Univariate Analysis } \\
\hline Factor & OR [95\%CI] & $p$ \\
\hline Albumin & $33.18[5.00-220.4]$ & $<0.001$ \\
\hline Cm & $10.76[2.586-44.78]$ & $<0.001$ \\
\hline CRP & $1.10[1.030-1.700]$ & 0.007 \\
\hline FFM & $1.05[1.0-1.111]$ & 0.040 \\
\hline Hemoglobin & $1.328[1.001-1.751]$ & 0.030 \\
\hline BMI & $1.11[1.01-1.210]$ & 0.025 \\
\hline Body weight & $1.04[1.008-1.078]$ & 0.013 \\
\hline & Multivariate Analysis & \\
\hline Factor & OR [95\%CI] & 0.009 \\
\hline Albumin & $50.48[2.594-182.39]$ & 0.007 \\
\hline Cm & $34.49[4.181-282.54]$ & 0.013 \\
\hline Irisin & $1.390[1.07-1.079]$ & \\
\hline
\end{tabular}

Finally, we also estimated diagnostic accuracy of irisin, inflammatory markers and $\mathrm{Cm}$ for distinguishing between cachectic and non-cachectic CHF patients. Among the studied factors, the strongest individual diagnostic accuracy reflected by AUC was achieved by $\mathrm{Cm}$ (AUC $=0.787$; sensitivity and specificity: $52.9 \%$ and $90.6 \%$, respectively). Notably, we observed improvement of the diagnostic power of the designed test combining at least two markers. The highest accuracy was demonstrated by test based on the combination of $\mathrm{Cm}$ with albumin (AUC $=0.917$ ). Combined analysis of $\mathrm{Cm}, \mathrm{CRP}$ and albumin demonstrated especially high diagnostic sensitivity of $93.3 \%$ with a specificity of $85.3 \%(A U C=0.929)$ (Figure $2 \mathrm{~A}$ ), however, the combination of $\mathrm{Cm}, \mathrm{CRP}$, albumin and irisin achieved a reduction in the sensitivity to $80 \%$, but the considerable specificity of $97.1 \%$ was demonstrated (AUC $=0.949$ ) (Figure $2 \mathrm{~B}$ ) as distinguishing between cachectic and non-cachectic CHF patients.

$\mathbf{A}$

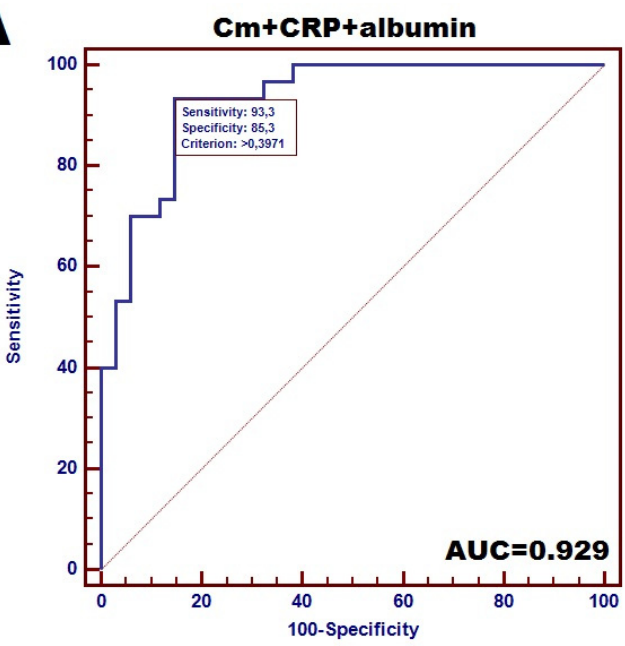

B

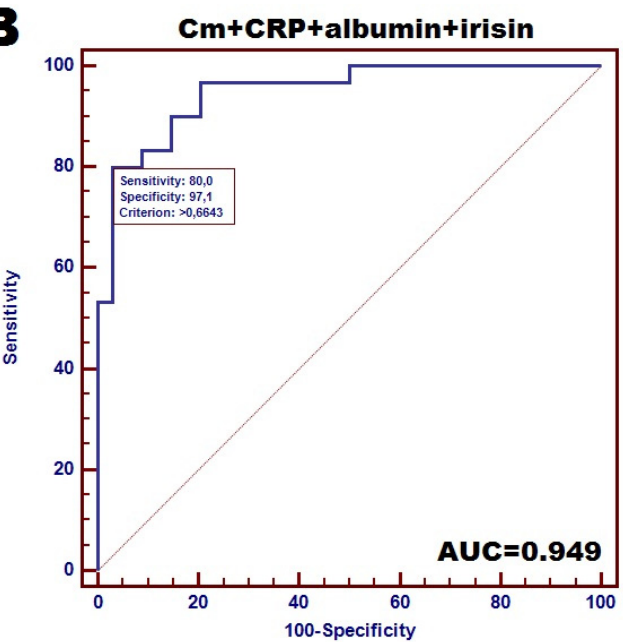

Figure 2. Accuracy of designed tests for distinguishing between cachectic and non-cachectic $\mathrm{CHF}$ female patients: (A) - Receiver operating curves (ROC) for combined analysis of 3 markers (Cm, CRP and albumin), (B)-ROC for 4 markers-based tests (combination of CM, CRP, albumin and irisin). 
Diagnostic accuracy of particular markers is demonstrated in the Table 5.

Table 5. Tests accuracy of CRP, irisin, albumin, $\mathrm{Cm}$ and their combination to distinguish cachectic $\mathrm{CHF}$ patients from non-cachectic $\mathrm{CHF}$ individuals.

\begin{tabular}{cccccc}
\hline Factor & Sensitivity & Specificity & AUC [95\%CI] & Cut-Off Value & $p$ \\
\hline Albumin & $93.5 \%$ & $52.8 \%$ & $0.724[0.582-0.788]$ & 3.30 & $<0.001$ \\
\hline CRP & $55.9 \%$ & $87.1 \%$ & $0.704[0.577-0.810]$ & 9.65 & 0.003 \\
\hline Cm & $52.9 \%$ & $90.6 \%$ & $0.787[0.669-0.878]$ & 0.789 & $<0.001$ \\
\hline Irisin & $64.7 \%$ & $46.9 \%$ & $0.580[0.452-0.700]$ & 8.11 & 0.270 \\
\hline Cm+CRP & $70.6 \%$ & $83.9 \%$ & $0.855[0.745-0.930]$ & - & $<0.001$ \\
\hline Cm+albumin & $96.8 \%$ & $76.5 \%$ & $0.917[0.821-0.971]$ & - & $<0.001$ \\
\hline Cm+Irisin & $61.8 \%$ & $96.9 \%$ & $0.849[0.740-0.925]$ & - & $<0.001$ \\
\hline CRP+albumin & $96.7 \%$ & $70.6 \%$ & $0.858[0.748-0.933]$ & - & $<0.001$ \\
\hline CRP+Irisin & $58.8 \%$ & $90.3 \%$ & $0.731[0.607-0.834]$ & - & $<0.001$ \\
\hline Cm+albumin+CRP & $93.3 \%$ & $85.3 \%$ & $0.929[0.938-0.979]$ & - & $<0.001$ \\
\hline 4 markers & $80 \%$ & $97.1 \%$ & $0.949[0.863-0.988]$ & - & $<0.001$ \\
\hline
\end{tabular}

\section{Discussion}

According to the literature, there are no reports of studies combining the use of biochemical markers and BIA in the diagnosis of cachexia in patients with CHF. Based on the literature findings, laboratory parameters have indicated that irisin is a clinically interesting adipomyokine reflecting cardiovascular system efficiency. The reason why irisin was chosen for our research are briefly presented below.

El-Mottaleb NA et al. found irisin to be a useful biomarker in the diagnosis of myocardial infarction with or without heart failure [9]. The authors evaluated eighty-six subjects (33 patients with myocardial infarction, 33 patients with myocardial infarction and heart failure, and 20 controls) and found negative correlations between irisin and BMI, WHR, SBP, DBP, troponin-I, CK-MB, TNF- $\alpha$, TC, TGs, and LDL-C. However, a positive association was observed between irisin and LVEF and HDL-C. Another study by Hsieh IC et al. showed that serum concentration of irisin might be a useful marker in STEMI monitoring. The level of this adipomyokineis elevated in post-STEMI patients with increased risk for adverse cardiovascular events. That is why the authors concluded that therapies targeting irisin may represent a new direction in future treatment [16]. A study by Silvestrini A et al. found higher level of irisin in HFpEF than in HFrEF patients ( $7.72 \pm 0.76$ vs. $2.77 \pm 0.77 \mathrm{ng} / \mathrm{mL}$, respectively). The authors correlated these findings with total antioxidant capacity (TAC), as an index of oxidative stress, and found an inverse correlation between irisin and TAC in HFpEF but not in HFrEF, which led them to conclude that different pathophysiological mechanisms are involved in the two CHF subtypes, and that oxidative stress modulates irisin secretion [8]. According to recent findings, circulating adropin is also an independent risk factor for heart disease, and its plasma level increased with the severity of HF. Interestingly, the study by Kalkan AK et al. evaluated adropin and irisin levels in cachectic and non-cachectic subjects, and the relationships between the levels of these proteins and clinical and laboratory parameters in patients with HFrEF. Cachectic patients $(n=44$, mean age: $65.4 \pm 11.2 ; 61.4 \%$ men) were identified in the study group of 86 patients. Serum irisin level was significantly higher in the cachexia group than in the controls (Irisin $(\mu \mathrm{g} / \mathrm{mL}) ; 2.6$ (IQR:2.2-4.4) vs. 2.1 (IQR:1.8-2.4); $p=0.001$ ), and positively correlated with BNP levels and NYHA class, and negatively correlated with BMI and serum albumin level (all $p$ values: $<0.001$ ) [5]. The results suggest that adropin and irisin may be novel markers of cardiac cachexia in patients with heart failure with a reduced ejection fraction.

It is worth mentioning that alterations in body composition, like the loss of skeletal muscle mass (sarcopenia or/and cachexia) or fat mass, are frequent in heart insufficiency. The prevalence and clinical 
consequences are very often underestimated. The assessment of cachexia among those patients has been a great challenge, as researchers use many different criteria of cachexia. Due to this fact, it has been difficult to make solid conclusions for further treatment strategies.

Our group was divided into cachectic and non-cachectic subjects, according to the criteria proposed by Evans WJ et al. These criteria include laboratory, clinical and functional parameters like: weight loss of at least $5 \%$ or more in 12 months or less in the presence of underlying illness, plus three of the following criteria: decreased muscle strength, fatigue, anorexia, low fat-free mass index, abnormal biochemistry (increased level of inflammatory markers: C-reactive protein $>5.0 \mathrm{mg} / \mathrm{L}$ ), IL-6 $>4.0 \mathrm{pg} / \mathrm{mL})$, anaemia $(<12 \mathrm{~g} / \mathrm{dL})$ and low concentration of serum albumin $(<3.2 \mathrm{~g} / \mathrm{dL})$.

In our research, CHF female patients who suffered from cachexia exhibited a significantly poorer general condition reflected by BIA, cardiac and laboratory parameters, compared to non-cachectic individuals. Regarding cardiac parameters, $61.8 \%$ cachectic women and $34.4 \%$ non-cachectic females were qualified to NYHA III and IV class $(p=0.030)$. The cachectic patients demonstrated a significant reduction of cardiac performance measured by $\mathrm{EF} \%(p=0.039)$.

According to recent findings, irisin is considered as an anti-inflammatory myokine against the pro-inflammatory activation of adipocytes, and is assumed to serve as a putative agent eliciting cardioprotection. Our results should comply with the above-mentioned observations. First, we noted significantly lower level of circulating irisin in cachectic CHF patients compared to non-cachectic study participants (median: $7.12 \mu \mathrm{g} / \mathrm{mL}$ vs. $7.61 \mu \mathrm{g} / \mathrm{mL} ; p=0.022$ ). This result is different from that reported by Kalkan et al. [5]. The reason for this might be the group of patients (our group consisted only of women, whereas the a above-mentioned study included both women and men), different criteria for the cachexia assessment (in our research we used the ones proposed by Evans et al. [15] whereas in Kalkan study the ones applied by $[17,18])$. Second, the significant negative correlation between plasma irisin concentration and the level of both CRP and TNF- $\alpha$ was recorded $(R=-0.362$ and $R=-0.243$; $p=0.004$ and $p=0.044$, respectively). Perhaps this can explain the more favourable cardiac and nutritional condition of non-cachectic patients. They had reduced inflammatory response represented by CRP and TNF- $\alpha$. This finding seems to be supported by the positive correlation between irisn concentration and $\mathrm{EF} \%$, as well as the negative correlation with NT-proBNP level $(R=0.253$ and $R=-0.320 ; p=0.046$ and $p=0.010$, respectively). Moreover, patients with lower irisin concentration had higher $\mathrm{Cm}$ values $(R=-0.393 ; p=0.005)$, however, CHF patients suffering from cachexia had lower levels of $\mathrm{Cm}(p<0.001)$. Interestingly, despite this non-pairability, both the low level of circulating irisin and $\mathrm{Cm}$ were selected as unfavourable factors affecting cachexia in CHF patients ( $\mathrm{OR}=1.39$ and 34.49, respectively). We also estimated diagnostic accuracy of irisin and $\mathrm{Cm}$ for distinguishing between CHF patients with the presence of cachexia and non-cachectic individuals. Combination of $\mathrm{Cm}$ and irisin with CRP and albumin concentration demonstrated considerable diagnostic accuracy for distinguishing between cachectic and non-cachectic CHF patients (sensitivity of $93.3 \%$ and specificity of $85.3 \%$; AUC $=0.949$ ).

Among studies assessing the impact of wasting syndromes (cachexia and sarcopenia) in HF on functional parameters, it is worth mentioning one recently published by Emani et al. [19]. In a group of 207 ambulatory male patients with clinically stable CHF, cachexia was present in $39(18.8 \%)$ of 207 patients, 14 of whom also fulfilled the characteristics of sarcopenia (sarcopenia + cachexia group, $6.7 \%$ ). Patients with sarcopenia were weaker and had a lower exercise capacity than both the patients without wasting syndromes and cachectic subjects in the CHF group. Handgrip strength, quadriceps strength, peak oxygen uptake (VO2), distance in the 6-minute walk test (6MWT) and quality of life (QoL) results were the lowest in the sarcopenia + cachexia group vs. the no wasting syndrome group $(p<0.05$ for all). This shows that loss of muscle with or without weight loss appears to have a pronounced influence on functional parameters like handgrip strength, quadriceps strength, VO2, 6MWT and QoL. Recently, a new class of biomarkers, circulating microRNA, is emerging, as they may provide additional pathophysiological information, helping to improve prognostic assessment. A number of circulating microRNAs are known to be altered in cachexia and sarcopenia $[20,21]$. Interestingly, among screened 
microRNAs, several are also candidate markers in heart failure [22,23], as well as some cachexia-related circulating microRNAs, such as microRNA-21 and microRNA-133, and are promising biomarkers in heart failure thatmight be able to help distinguish underlying etiology, including cachexia [24].

Unfortunately, our group was focused only on cachectic state without including sarcopenia impact, which is, of course, one of the limitations of the study. Additionally, the study includes only females, and this is another limitation of the research, which reflects the presence of a selection bias. However, our findings might be useful for clinical practice in distinguishing cachectic patients with CHF (by measuring Cm by BIA and laboratory parameters- irisin, albumin, CRP) in order to monitor these patients more accurately, as the diagnostic accuracy is very high (sensitivity of $93.3 \%$ and specificity of $85.3 \%$; AUC $=0.929$ ).

\section{Conclusions}

There are neither established criteria nor clinical guidelines allowing early detection and management of CC. However, both the high prevalence of cachexia among CHF individuals and serious conditions affected by this multifactorial syndrome, encourage facing this clinical problem with an interdisciplinary approach involving the analysis of different biomarkers. Our findings demonstrate novel perspectives for cachectic CHF patients, which include the following prospective benefits: selection of CC risk group, prediction of treatment outcomes and disease course. Our approach has not been practiced before, but we believe that combined analysis of at least a few non-invasive markers, such as irisin, $\mathrm{Cm}$, albumin and inflammatory markers, could improve clinical opportunities for CC management. However, the putative clinical utility of the proposed diagnostic procedure needs to be confirmed by studies involving a larger number of enrolled cases.

Author Contributions: Idea of the study T.M.-M., G.S.; Sample collection: G.S., A.S.-D.; Biochemical experiments: M.M.; Statistical analysis and data interpretation: T.P.; manuscript writing and editing: G.S., T.M.-M., T.P. All authors have read and agreed to the published version of the manuscript.

Conflicts of Interest: The authors declare no conflict of interest.

\section{References}

1. Sobieszek, G.; Mlak, R.; Skwarek-Dziekanowska, A.; Jurzak-Myśliwy, A.; Homa-Mlak, I.; Małecka-Massalska, T. Electrical Changes in Polish Patients with Chronic Heart Failure: Preliminary Observations. Medicina 2019, 55, 484. [CrossRef] [PubMed]

2. Ramani, G.V.; Uber, P.A.; Mehra, M.R. Chronic Heart Failure: Contemporary Diagnosis and Management. Mayo Clin. Proc. 2010, 85, 180-195. [CrossRef] [PubMed]

3. Gřiva, M. Cardiac cachexia-Up-to-date 2015. Cor Vasa 2016, 58. [CrossRef]

4. Okoshi, M.P.; Capalbo, R.V.; Romeiro, F.G.; Okoshi, K. Cardiac Cachexia: Perspectives for Prevention and Treatment. Arq. Bras. Cardiol. 2016. [CrossRef] [PubMed]

5. Kalkan, A.K.; Cakmak, H.A.; Erturk, M.; Kalkan, K.U.; Uzun, F.; Tasbulak, O.; Diker, V.O.; Aydin, S.; Celik, A. Adropin and Irisin in Patients with Cardiac Cachexia. Arq. Bras. Cardiol. 2018. [CrossRef]

6. Aydin, S.; Aydin, S. Irisin Concentrations as a Myocardial Biomarker. Biomark. Cardiovasc. Dis. 2016, 489-504. [CrossRef]

7. Piatek, P.; Fabian-Danielewska, A.; Korabiusz, K. The role of irisin in ischemic heart diesease. J. Educ. Health Sport 2019, 9, 30-35. [CrossRef]

8. Silvestrini, A.; Bruno, C.; Vergani, E.; Venuti, A.; Favuzzi, A.M.R.; Guidi, F.; Nicolotti, N.; Meucci, E.; Mordente, A.; Mancini, A. Circulating irisin levels in heart failure with preserved or reduced ejection fraction: A pilot study. PLoS ONE 2019, 14. [CrossRef]

9. El-Mottaleb, N.A.A.; Galal, H.M.; Maghraby, K.M.E.; Gadallah, A.I. Serum irisin level in myocardial infarction patients with or without heart failure. Can. J. Physiol. Pharmacol. 2019, 97, 932-938. [CrossRef]

10. Khalil, S.; Mohktar, M.; Ibrahim, F. The Theory and Fundamentals of Bioimpedance Analysis in Clinical Status Monitoring and Diagnosis of Diseases. Sensors 2014, 14, 10895-10928. [CrossRef] 
11. Grundmann, O.; Yoon, S.L.; Williams, J.J. The value of bioelectrical impedance analysis and phase angle in the evaluation of malnutrition and quality of life in cancer patients-A comprehensive review. Eur. J. Clin. Nutr. 2015, 69, 1290-1297. [CrossRef] [PubMed]

12. Génot, N.; Mewton, N.; Bresson, D.; Zouaghi, O.; Francois, L.; Delwarde, B.; Kirkorian, G.; Bonnefoy-Cudraz, E. Bioelectrical impedance analysis for heart failure diagnosis in the ED. Am. J. Emerg. Med. 2015, 33, 1025-1029. [CrossRef] [PubMed]

13. Ponikowski, P.; Voors, A.A.; Anker, S.D.; Bueno, H.; Cleland, J.G.F.; Coats, A.J.S.; Falk, V.; González-Juanatey, J.R.; Harjola, V.P.; Jankowska, E.A.; et al. 2016 ESC Guidelines for the diagnosis and treatment of acute and chronic heart failure. Eur. Heart J. 2016, 37, 2129-2200. [CrossRef] [PubMed]

14. The Criteria Committee of the New York Heart Association. Nomenclature and Criteria for Diagnosis of Diseases of the Heart and Great Vessels, 9th ed.; Little, Brown \& Co.: Boston, MA, USA, 1994; pp. 253-256.

15. Evans, W.J.; Morley, J.E.; Argiles, J.; Bales, C.; Baracos, V.; Guttridge, D.; Jatoi, A.; Kalantar-Zadeh, K.; Lochs, H.; Mantovani, G.; et al. Cachexia: A new definition. Clin. Nutr. 2008, 27, 793-799. [CrossRef]

16. Hsieh, I.C.; Ho, M.Y.; Wen, M.S.; Chen, C.C.; Hsieh, M.J.; Lin, C.P.; Yeh, J.K.; Tsai, M.L.; Yang, C.H.; Wu, V.C.; et al. Serum irisin levels are associated with adverse cardiovascular outcomes in patients with acute myocardial infarction. Int. J. Cardiol. 2018, 261, 12-17. [CrossRef] [PubMed]

17. McMurray, J.J.; Adamopoulos, S.; Anker, S.D.; Auricchio, A.; Böhm, M.; Dickstein, K.; Falk, V.; Filippatos, G.; Fonseca, C.; Gomez-Sanchez, M.A.; et al. ESC Guidelines for the diagnosis and treatment of acute and chronic heart failure 2012: The Task Force for the Diagnosis and Treatment of Acute and Chronic Heart Failure 2012 of the European Society of Cardiology. Developed in collaboration with the Heart Failure Association (HFA) of the ESC. Eur. J. Heart Fail. 2012, 14, 803-869. [CrossRef]

18. Okoshi, M.P.; Romeiro, F.G.; Paiva, S.A.R.; Okoshi, K. Heart Failure-Induced Cachexia. Arq. Bras. Cardiol. 2013. [CrossRef]

19. Emami, A.; Saitoh, M.; Valentova, M.; Sandek, A.; Evertz, R.; Ebner, N.; Loncar, G.; Springer, J.; Doehner, W.; Lainscak, M.; et al. Comparison of sarcopenia and cachexia in men with chronic heart failure: Results from the Studies Investigating Co-morbidities Aggravating Heart Failure (SICA-HF). Eur. J. Heart Fail. 2018, 20, 1580-1587. [CrossRef]

20. Di Ieva, A.; Butz, H.; Niamah, M.; Rotondo, F.; De Rosa, S.; Sav, A.; Yousef, G.M.; Kovacs, K.; Cusimano, M.D. MicroRNAs as biomarkers in pituitary tumors. Neurosurgery 2014, 75, 181-189. [CrossRef]

21. Okugawa, Y.; Yao, L.; Toiyama, Y.; Yamamoto, A.; Shigemori, T.; Yin, C.; Omura, Y.; Ide, S.; Kitajima, T.; Shimura, T.; et al. Prognostic impact of sarcopenia and its correlation with circulating miR-21 in colorectal cancer patients. Oncol. Rep. 2018, 39, 1555-1564. [CrossRef]

22. De Rosa, S.; Indolfi, C. Circulating microRNAs as Biomarkers in Cardiovascular Diseases. In Circulating microRNAs in Disease Diagnostics and Their Potential Biological Relevance; Experientia, S., Igaz, P., Eds.; Springer: Basel, Switzerland, 2015; Volume 106.

23. Antunes-Correa, L.M.; Trevizan, P.F.; Bacurau, A.V.N.; Ferreira-Santos, L.; Gomes, J.L.P.; Urias, U.; Oliveira, P.A.; Alves, M.J.N.N.; de Almeida, D.R.; Brum, P.C.; et al. Effects of aerobic and inspiratory training on skeletal muscle microRNA-1 and downstream-associated pathways in patients with heart failure. J. Cachexia Sarcopenia Muscle 2020, 11, 89-102. [CrossRef] [PubMed]

24. De Rosa, S.; Eposito, F.; Carella, C.; Strangio, A.; Ammirati, G.; Sabatino, J.; Abbate, F.G.; Iaconetti, C.; Liguori, V.; Pergola, V.; et al. Transcoronary concentration gradients of circulating microRNAs in heart failure. Eur. J. Heart Fail. 2018, 20, 1000-1010. [CrossRef] [PubMed]

(C) 2020 by the authors. Licensee MDPI, Basel, Switzerland. This article is an open access article distributed under the terms and conditions of the Creative Commons Attribution (CC BY) license (http://creativecommons.org/licenses/by/4.0/). 